Bundesgesundheitsbl 2016 · 59:1028-1039

DOI 10.1007/s00103-016-2388-6

C) Springer-Verlag Berlin Heidelberg 2016
Bekanntmachung des Umweltbundesamtes

\section{Zur Frage eines Asthma auslösenden bzw. verschlechternden Potenzials von Formaldehyd in der Innenraumluft bei Kindern}

\author{
Mitteilung des Ausschusses für Innenraumrichtwerte (AIR)
}

\section{Einleitung}

Als Hauptwirkung eingeatmeten Formaldehyds wird allgemein die Reizwirkung auf den oberen Atemtrakt angesehen. Bei chronischer Einwirkung können sehr hohe Formaldehyd-Konzentrationen zytotoxische und kanzerogene Wirkungen in diesem Gewebe nach sich ziehen [1]. Während bei Ratten Formaldehyd fast ausschließlich in der Nase resorbiert wird (zu $99 \%$ bei $0,1-1 \mathrm{mg} / \mathrm{m}^{3}$ ), wird es nach Modellabschätzungen im gleichen Konzentrationsbereich bei Primaten und Menschen nur zu $87 \%$ bzw. zu $85 \%$ in der Nase, aber zu einem geringen Anteil auch im oberen Atemtrakt (Rachen, Luftröhre und Bronchien) aufgenommen [2].

$\mathrm{Ob}$ eingeatmetes Formaldehyd auch in tiefere Teile des Atemtrakts gelangen und insbesondere bei Kindern Asthma auslösen oder verschlimmern kann, wird kontrovers diskutiert. Zur Ermittlung des Kenntnisstands hinsichtlich dieser Fragestellung wurde eine Recherche bei Pubmed, Web of Science und in Google mit den Stichworten "formaldehyde“, „asthma“, „allergy“ und „children“ und z. T. mit den deutschen Begriffen durchgeführt.

Von den gefundenen Studien wurden solche ohne Angaben zur Exposition gegenüber Formaldehyd in der Innenraumluft ausgeschlossen. Es verblieben etwa 90 Studien, die näher ausgewertet wurden.

\section{Ergebnisse von Studien zu Asthma nach inhalativer Formaldehyd-Exposition}

\section{Epidemiologische Studien an Formaldehyd-exponierten Kindern in Innenräumen}

Im Rahmen der Literaturrecherche ließen sich verschiedene epidemiologische Studien an Kindern nach einer Exposition gegenüber Formaldehyd in der Luft verschiedener Aufenthaltsräume mit unterschiedlichem Studiendesign finden. Bei ihnen wurde insbesondere mittels Fragebogen nach asthmabezogenen Symptomen wie keuchenden, pfeifenden Atemgeräuschen (im Englischen als „wheezing“ bezeichnet) oder nach einem ärztlich diagnostizierten Asthma gefragt. In der Regel handelte es sich um Selbsteinschätzungen bzw. Angaben der Eltern. Die gefundenen Studien und ihre wesentlichen Charakteristika und Ergebnisse, insbesondere hinsichtlich der Exposition gegenüber Formaldehyd in der Innenraumluft, sind in - Tab. 1 zusammengestellt.

Grundsätzlich muss angemerkt werden, dass in epidemiologischen Studien der komplexen Expositionssituation von Kindern oft nur begrenzt nachgegangen werden kann. So liegen in der Regel nur Messergebnisse aus einzelnen Umweltkompartimenten, in denen sich Kinder aufhalten (z. B. in Schulen oder Wohnräumen), vor oder es handelt sich um Kurzzeitmessungen. Andere Substanzen in In- nenräumen, für die es Hinweise gibt, dass sie an der Entstehung bzw. Verstärkung des Asthmas beteiligt sein könnten, wurden oft nicht gemessen. Auf die grundsätzlichen Schwierigkeiten bei der Erhebung der Diagnose Asthma in epidemiologischen Studien im Kleinkindalter wird in einem späteren Abschnitt eingegangen.

\section{Interventionsstudien}

Bisher ist in der Literatur nur eine Interventionsstudie im Kindesalter publiziert, die auch Messungen der Formaldehyd-Konzentration in der Innenraumluft umfasste [3]. In ihr wurden 83 asthmatische Kinder im Alter von 3-12 Jahren, die in schlecht belüfteten Wohnräumen (Lüftungsraten unter $0,3 \mathrm{~h}^{-1}$, im Mittel $0,18 \mathrm{~h}^{-1}$ ) lebten, über 2 Jahre verfolgt. Nach dem ersten Jahr wurde in den Wohnräumen von 43 Kindern durch Lüftungssysteme die Luftwechselrate um $0,15 \mathrm{~h}^{-1}$ gezielt erhöht. Diese Maßnahme führte zu einer statistisch signifikanten Verringerung der relativen Luftfeuchte, an ausgewählten flüchtigen organischen Verbindungen, an Formaldehyd sowie an Schimmelpilzsporen in der Innenraumluft. In gesundheitlicher Hinsicht wurden bei der primären Zielvariablen „Anzahl der Tage mit Symptomen“ keine Unterschiede zwischen den Gruppen beobachtet. Aber in der Interventionsgruppe wurde eine verminderte Häufigkeit folgender sekundärer Zielvariablen gefunden: mindestens eine Wheezing-Episode im Jahr zu ha- 
Tab. 1 Studien zum Zusammenhang von Formaldehyd (FA) in der Innenraumluft und Asthma bzW. asthmabezogenen Symptomen bei Kindern

\begin{tabular}{|c|c|c|c|c|c|c|c|}
\hline Referenz & Ort & $\mathrm{N}$ & Alter (Jahre) & $\begin{array}{l}\text { Informations- } \\
\text { quellen }\end{array}$ & Exposition & Adjustierungen & Ergebnisse \\
\hline \multicolumn{8}{|c|}{ Interventionsstudien } \\
\hline $\begin{array}{l}\text { Lajoie et al. } \\
2015 \text { [3] }\end{array}$ & $\begin{array}{l}\text { Kanada, } \\
2008-11\end{array}$ & $\begin{array}{l}43 \text { in } \\
\text { Interventi- } \\
\text { onsgruppe } \\
\text { und } 39 \\
\text { Kontrollen }\end{array}$ & $3-12$ & Ärztliche Diagnose & $\begin{array}{l}\text { Passivsammler, HPLC, } \\
\text { Schlafzimmer, } 6-8 \\
\text { Tage; AM: } 37 \text { vor } \\
\text { und } 24 \mu \mathrm{g} / \mathrm{m}^{3} \text { nach } \\
\text { Intervention (Winter) } \\
\text { und } 57 \text { und } 47 \mu \mathrm{g} / \mathrm{m}^{3} \\
\text { (Sommer) }\end{array}$ & $\begin{array}{l}\text { Alter, Geschlecht, } \\
\text { Ekzem Vorgeschichte, } \\
\text { elterliche Bildung }\end{array}$ & $\begin{array}{l}\text { Sign. Assoziation zwi- } \\
\text { schen FA-Reduktion und } \\
\text { jährliche Prävalenz von } \\
\geq 1 \text { Wheezing-Episode, } \\
\text { nächtlicher Husten und } \\
\geq 1 \text { Besuch einer Notfall- } \\
\text { station }\end{array}$ \\
\hline \multicolumn{8}{|c|}{ Fall-Kontroll-Studien } \\
\hline $\begin{array}{l}\text { Rumchev et } \\
\text { al. } 2002 \text { [12] }\end{array}$ & $\begin{array}{l}\text { Australia, } \\
\text { 1997-99 }\end{array}$ & $\begin{array}{l}88 \text { Fälle } \\
\text { und } 104 \\
\text { Kontrollen }\end{array}$ & $0,5-3$ & $\begin{array}{l}\text { Elternfragebogen, } \\
\text { Pricktest }\end{array}$ & $\begin{array}{l}\text { Passivsammler, } \\
\text { DNPH, Sommer und } \\
\text { Winter, Wohn- und } \\
\text { Schlafraum über } 8 \mathrm{~h} \text {; } \\
\text { AM: } 28-30 \mu \mathrm{g} / \mathrm{m}^{3}, \\
\text { Max: } 190-224 \mu \mathrm{g} / \mathrm{m}^{3}\end{array}$ & $\begin{array}{l}\text { Allergenlevel, Klima, } \\
\text { Atopie, familiäres } \\
\text { Asthma, Alter, Ge- } \\
\text { schlecht, Rauchen, } \\
\text { Sozialstatus, Gashei- } \\
\text { zung }\end{array}$ & $\begin{array}{l}\text { Asthmarisiko per } 10 \mu \mathrm{g} / \\
\mathrm{m}^{3} \text {-Erhöhung: } \mathrm{OR}=1,03 \\
(95 \% \mathrm{Cl}: 1,02-1,04) \text {; } \\
\text { Fälle } 38 \mu \mathrm{g} / \mathrm{m}^{3} \text { vs. Kontrol- } \\
\text { len } 24 \mu \mathrm{g} / \mathrm{m}^{3}(p<0,001)\end{array}$ \\
\hline $\begin{array}{l}\text { Venn et al. } \\
2003[14]\end{array}$ & $\begin{array}{l}\text { England, } \\
1998 / 99\end{array}$ & $\begin{array}{l}193 \text { mit } \\
\text { persis- } \\
\text { tentem } \\
\text { Wheezing } \\
\text { und } 223 \\
\text { Kontrollen }\end{array}$ & $9-11$ & $\begin{array}{l}\text { Selbstberichteter } \\
\text { Fragebogen }\end{array}$ & $\begin{array}{l}\text { Passivsammler, } \mathrm{LC} \text {, } \\
\text { Schlafzimmer for } 3 \\
\text { Tage; } 0->32 \mu \mathrm{g} / \mathrm{m}^{3}\end{array}$ & Alter, Geschlecht & $\begin{array}{l}\text { Keine sign. Assoziation } \\
\text { zwischen Wheezing und } \\
\text { FA; berichtete nächtliche } \\
\text { Symptome sind sign. } \\
\text { assoziiert mit FA }\end{array}$ \\
\hline $\begin{array}{l}\text { Tavernier et } \\
\text { al. } 2006 \text { [15] }\end{array}$ & England & $\begin{array}{l}105 \text { Asth- } \\
\text { matiker, } 95 \\
\text { Kontrollen }\end{array}$ & $4-17$ & $\begin{array}{l}\text { Fragebogen und } \\
\text { ärztl. Diagnose }\end{array}$ & Zweimal, DNPH, HPLC & & $\begin{array}{l}\text { Keine Unterschiede in der } \\
\text { FA-Exposition zwischen- } \\
\text { Fällen und Kontrollen }\end{array}$ \\
\hline $\begin{array}{l}\text { Hulin et al. } \\
2010 \text { [17] }\end{array}$ & Frankreich & $\begin{array}{l}56 \text { Asthma- } \\
\text { tiker und } 58 \\
\text { Kontrollen }\end{array}$ & $12,6(\mathrm{AM})$ & ISAAC Fragebogen & $\begin{array}{l}\text { Passivsammler, } 1 \\
\text { Woche, Wohnraum; } \\
4-75 \mu \mathrm{g} / \mathrm{m}^{3}\end{array}$ & $\begin{array}{l}\text { Alter, Geschlecht, } \\
\text { Familiäre Allergie, } \\
\text { Passivrauch }\end{array}$ & $\begin{array}{l}\text { Nur in ländlichem Gebiet } \\
\text { sign. Assoziation zum } \\
\text { lebenslangen, nicht } \\
\text { aber aktuellem Asthma: } \\
\text { OR=10,7 }(95 \% \text { Cl: } \\
1,67-67,6)\end{array}$ \\
\hline $\begin{array}{l}\text { Hwang et al. } \\
2011 \text { [16] }\end{array}$ & Korea, 2008 & $\begin{array}{l}33 \text { Asthma- } \\
\text { tiker und } 40 \\
\text { Kontrollen }\end{array}$ & $8-13$ & Ärztliche Diagnose & $\begin{array}{l}\text { Passivsammler, UV, } \\
\text { chlaf- und Wohnraum } \\
\text { über } 3 \text { Tage; } 31 \mu \mathrm{g} / \mathrm{m}^{3} \\
\text { (Fälle) and } 36 \mu \mathrm{g} / \mathrm{m}^{3} \\
\text { (Kontrollen) }\end{array}$ & $\begin{array}{l}\text { Familiäres Asthma, } \\
\text { Alter, Geschlecht, Pas- } \\
\text { sivrauch, Sozialstatus }\end{array}$ & $\begin{array}{l}\text { Keine sign. Unterschiede } \\
\text { zwischen den Gruppen }\end{array}$ \\
\hline $\begin{array}{l}\text { Hsu et al. } \\
2012[18]\end{array}$ & $\begin{array}{l}\text { Taiwan, } \\
2005 / 6\end{array}$ & $\begin{array}{l}59 \text { Fälle } \\
\text { and } 42 \text { Kon- } \\
\text { trollen }\end{array}$ & $3-9$ & $\begin{array}{l}\text { Elternfragebogen, } \\
\text { ärztliche Diagnose }\end{array}$ & $\begin{array}{l}\text { DNPH, HPLC-UV; } 24 \mathrm{~h}, \\
\text { Kinderzimmer; alle: } \\
8 \mu \mathrm{g} / \mathrm{m}^{3} \text { (Median) }\end{array}$ & & $\begin{array}{l}\text { Sign. niedrigere FA-Ge- } \\
\text { halte in Häusern mit } \\
\text { Asthmatikern gegenüber } \\
\text { den Kontrollen (Median: } \\
4 \text { vs. 14) }\end{array}$ \\
\hline \multicolumn{8}{|c|}{ Kohortenstudien } \\
\hline $\begin{array}{l}\text { Smedje \& } \\
\text { Norbäck } \\
2001[9]\end{array}$ & $\begin{array}{l}\text { Schweden, } \\
\text { 1993-95 }\end{array}$ & 1347 & Schulkinder & $\begin{array}{l}\text { Selbstberichteter } \\
\text { Fragebogen }\end{array}$ & $\begin{array}{l}\text { Zweimal } 1993 \text { und } \\
1995 \text { in } 39 \text { Schulen } \\
\text { (2-5 Klassenräume), } \\
4 \mathrm{~h} ; \mathrm{DNPH}, \mathrm{LC} ; \mathrm{AM:} 8 \text {, } \\
<5-72 \mu \mathrm{g} / \mathrm{m}^{3}\end{array}$ & $\begin{array}{l}\text { Geschlecht, Alter, } \\
\text { Rauchen, familiäre } \\
\text { Atopie }\end{array}$ & $\begin{array}{l}\text { OR Asthmainzidenz per } \\
10 \mu \mathrm{g} / \mathrm{m}^{3} \text { Erhöhung }=1,2 \\
(95 \% \mathrm{Cl}: 0,8-1,7), \text { aber } \\
\text { sign. für Katzenallergen } \\
\text { und Staub; Kinder ohne } \\
\text { Atopie sign. höhere } \\
\text { Inzidenz mit Asthmadi- } \\
\text { agnose:OR=1,7 ( } 95 \% \mathrm{Cl} \text { : } \\
\text { 1,1-2,6)] }\end{array}$ \\
\hline
\end{tabular}




\begin{tabular}{|c|c|c|c|c|c|c|c|}
\hline Referenz & Ort & $\mathbf{N}$ & Alter (Jahre) & $\begin{array}{l}\text { Informations- } \\
\text { quellen }\end{array}$ & Exposition & Adjustierungen & Ergebnisse \\
\hline $\begin{array}{l}\text { Raa- } \\
\text { schou-Ni- } \\
\text { elsen et al. } \\
2010 \text { [10] }\end{array}$ & Dänemark & 411 & $\begin{array}{l}\text { Während der } \\
\text { ersten } 18 \text { Le- } \\
\text { bensmonate }\end{array}$ & $\begin{array}{l}\text { Tägliches Whee- } \\
\text { zing-Tagebuch }\end{array}$ & $\begin{array}{l}\text { Passivsammler, drei- } \\
\text { mal je } 10 \text { Wochen, } \\
\text { Kinderschlafraum; } \\
\text { DNPH, HPLC-UV; } \\
\text { AM: } 20,3 \mu \mathrm{g} / \mathrm{m}^{3}, 95 \\
\text { Perzentil: } 37 \mu \mathrm{g} / \mathrm{m}^{3}\end{array}$ & $\begin{array}{l}\text { Geschlecht, Wohnort, } \\
\text { mütterliche Bildung, } \\
\text { Lungenfunktion }\end{array}$ & $\begin{array}{l}\text { Keine Assoziation zwi- } \\
\text { schen Wheezing und FA }\end{array}$ \\
\hline $\begin{array}{l}\text { Roda et al. } \\
2013 \text { [11] }\end{array}$ & $\begin{array}{l}\text { Frankreich, } \\
\text { 2003-06 }\end{array}$ & 3840 & $\begin{array}{l}\text { Während der } \\
\text { ersten } 12 \text { Le- } \\
\text { bensmonate }\end{array}$ & $\begin{array}{l}\text { Interviewerfrage- } \\
\text { bogen }\end{array}$ & $\begin{array}{l}\text { Passivsammler, } \\
\text { viermal über } 7 \text { Tage } \\
\text { in } 196 \text { Kinderschlaf- } \\
\text { räumen; HPLC-UV; } \\
\text { Berechnung der jähr- } \\
\text { lichen Mittelwerte } \\
\text { für alle Räume: Mittel } \\
19 \mu \mathrm{g} / \mathrm{m}^{3}\end{array}$ & $\begin{array}{l}\text { Geschlecht, } \\
\text { Sozialstatus, Stillen, } \\
\text { Passivrauch, Episoden } \\
\text { respiratorischer } \\
\text { Infekte }\end{array}$ & $\begin{array}{l}\text { OR pro } 10 \mu \mathrm{g} / \mathrm{m}^{3} \\
\text { Erhöhung von Whee- } \\
\text { zing }=1,45(95 \% \mathrm{Cl} \text { : } \\
1,08-1,96) \text {, aber nur bei } \\
\text { Kindern ohne familiäre } \\
\text { Allergiegeschichte }\end{array}$ \\
\hline \multicolumn{8}{|c|}{ Querschnittsstudien } \\
\hline $\begin{array}{l}\text { Krzyzanows- } \\
\text { ki et al. } 1990 \\
{[19]}\end{array}$ & USA & 298 & $6-15$ & $\begin{array}{l}\text { Selbstberichteter } \\
\text { Fragebogen }\end{array}$ & $\begin{array}{l}\text { Passivsammler, } 1 \\
\text { Woche, Küche, Wohn- } \\
\text { und Schlafraum; } \\
\text { NaHSO }_{3} \text {; Peakflow } \\
\text { viermal täglich; AM: } \\
33 \mu \mathrm{g} / \mathrm{m}^{3}, \text { ca. } 7 \% \\
>75 \mu \mathrm{g} / \mathrm{m}^{3}\end{array}$ & Keine Angaben & $\begin{array}{l}\text { Sign. höhere Prävalenz an } \\
\text { aktuellem Asthma bei Ge- } \\
\text { halten }>75 \mu \mathrm{g} / \mathrm{m}^{3} \text { (aber } \\
\text { nur bei Koexposition mit } \\
\text { Passivrauch); Peakflow } \\
\text { erniedrigt mit zunehmen- } \\
\text { der FA-Konzentration }\end{array}$ \\
\hline $\begin{array}{l}\text { Smedje et al. } \\
1997 \text { [20] }\end{array}$ & $\begin{array}{l}\text { Schweden, } \\
1992\end{array}$ & 627 & $13-14$ & $\begin{array}{l}\text { Selbstberichteter } \\
\text { Fragebogen }\end{array}$ & $\begin{array}{l}\text { Einmal im Klassen- } \\
\text { raum, DNPH über } 4 \mathrm{~h} \text {; } \\
<5-10 \mu \mathrm{g} / \mathrm{m}^{3}\end{array}$ & & $\begin{array}{l}\text { Sign. Assoziation zwi- } \\
\text { schen aktuellem Asthma } \\
\text { und FA-Gehalten:OR=1,1 } \\
(95 \% \mathrm{Cl}: 1,01-1,2)\end{array}$ \\
\hline $\begin{array}{l}\text { Garrett et al. } \\
1999[21]\end{array}$ & $\begin{array}{l}\text { Australien, } \\
1994 / 5\end{array}$ & 148 & $7-14$ & $\begin{array}{l}\text { Elterlicher Frage- } \\
\text { bogen }\end{array}$ & $\begin{array}{l}\text { Passivsammler, } 80 \\
\text { Wohnungen; viermal } \\
\text { über ein Jahr je } 4 \\
\text { Tage, Schlaf- und } \\
\text { Wohnraum, Küche; } \\
\text { DNPH, LC-UV; Medi- } \\
\text { an: } 16 \mu \mathrm{g} / \mathrm{m}^{3} \text {, Max: } \\
139 \mu \mathrm{g} / \mathrm{m}^{3}\end{array}$ & $\begin{array}{l}\text { Elterliches Asth- } \\
\text { ma, Geschlecht, } \\
\text { Haustiere, NOx, } \\
\text { Schimmelpilzsporen, } \\
\text { Milbenallergene }\end{array}$ & $\begin{array}{l}\text { Kein sign. OR für Atopie je } \\
10 \mu \mathrm{g} / \mathrm{m}^{3} \text { Erhöhung } 1,40 \\
(95 \% \mathrm{Cl}: 0,98-2,00) \text { nach } \\
\text { Adjustierung }\end{array}$ \\
\hline $\begin{array}{l}\text { Jaakola et al. } \\
2004 \text { [23] }\end{array}$ & $\begin{array}{l}\text { Russland } \\
\text { 1996/7 }\end{array}$ & 5951 & $8-12$ & $\begin{array}{l}\text { Selbstberichteter } \\
\text { und Elternfrage- } \\
\text { bogen }\end{array}$ & $\begin{array}{l}\text { Keine Messungen, } \\
\text { Bericht über Einrich- } \\
\text { tungsmaterialien }\end{array}$ & $\begin{array}{l}\text { Alter, Geschlecht, } \\
\text { Frühgeburt, geringes } \\
\text { Geburtsgewicht, } \\
\text { elterliche Atopie, } \\
\text { elterliche Bildung, } \\
\text { Passivrauch }\end{array}$ & $\begin{array}{l}\text { Sign. Assoziation zwi- } \\
\text { schen Spanplatteneinsatz } \\
\text { und aktuellem Whee- } \\
\text { zing: OR=1,39 ( } 95 \% \mathrm{Cl} \text { : } \\
\text { 1,07-1,80), aber nicht mit } \\
\text { aktuellem Asthma }\end{array}$ \\
\hline $\begin{array}{l}\text { Mi et al. } \\
2006[24]\end{array}$ & China, 2000 & 1414 & $13-14$ & $\begin{array}{l}\text { Selbstberichteter } \\
\text { Fragebogen }\end{array}$ & $\begin{array}{l}\text { DNPH, } 4 \text { Stdn., } 10 \\
\text { Schulen; } 3-20 \mu \mathrm{g} / \mathrm{m}^{3}\end{array}$ & $\begin{array}{l}\text { Alter, Geschlecht, } \\
\text { Rauchen, Schimmel }\end{array}$ & $\begin{array}{l}\text { Keine sign. Assoziation } \\
\text { mit aktuellem Wheezing, } \\
\text { Atemnot, Asthmaatta- } \\
\text { cken und Medikation, } \\
\text { und aktuellem Asthma }\end{array}$ \\
\hline $\begin{array}{l}\text { Zhao et al. } \\
2008 \text { [25] }\end{array}$ & China, 2004 & 1480 & $11-15$ & $\begin{array}{l}\text { ISAAC questi- } \\
\text { onnaire }\end{array}$ & $\begin{array}{l}\text { In ausgewählten } \\
\text { Klassenräumen von } \\
10 \text { Schulen, Passiv- } \\
\text { sammler über } 7 \text { Tage, } \\
\text { k. A. Messverfahren; } \\
1-5 \mu \mathrm{g} / \mathrm{m}^{3}\end{array}$ & $\begin{array}{l}\text { Hierarchisches Model, } \\
\text { Clustereffekte }\end{array}$ & $\begin{array}{l}\text { Keine sign. Assoziatio- } \\
\text { nen mit Wheezing und } \\
\text { Atemlosigkeit }\end{array}$ \\
\hline $\begin{array}{l}\text { Kim et al. } \\
2011 \text { [26] }\end{array}$ & Korea, 2004 & 1028 & 10 (AM) & $\begin{array}{l}\text { Selbstberichteter } \\
\text { Fragebogen und } \\
\text { ärztliche Diagnose }\end{array}$ & $\begin{array}{l}12 \text { Schulen, Passiv- } \\
\text { sammler über } 7 \text { Tage, } \\
\text { k. A. Messverfahren; } \\
16-47 \mu \mathrm{g} / \mathrm{m}^{3}\end{array}$ & $\begin{array}{l}\text { Alter, Geschlecht, } \\
\text { Haustiere, Passiv- } \\
\text { rauch, Feuchteschä- } \\
\text { den }\end{array}$ & $\begin{array}{l}\text { Keine sign. Assoziation } \\
\text { zwischen aktuellem } \\
\text { Asthma und ärztlicher } \\
\text { Asthmadiagnose und FA }\end{array}$ \\
\hline
\end{tabular}




\begin{tabular}{|c|c|c|c|c|c|c|c|}
\hline Referenz & Ort & $\mathbf{N}$ & Alter (Jahre) & $\begin{array}{l}\text { Informations- } \\
\text { quellen }\end{array}$ & Exposition & Adjustierungen & Ergebnisse \\
\hline $\begin{array}{l}\text { Martins et al. } \\
2012 \text { [27] }\end{array}$ & $\begin{array}{l}\text { Portugal, } \\
2006 / 7\end{array}$ & $\begin{array}{l}51 \text { (mit } \\
\text { Weezing) }\end{array}$ & $7(\mathrm{AM})$ & $\begin{array}{l}\text { Elternfragebogen, } \\
\text { Lungenfunktions- } \\
\text { prüfung, eNO }\end{array}$ & $\begin{array}{l}\text { Passivsammler, } \\
\text { einmal in Schule } \\
\text { und Schlafraum; } \\
\text { k. A. Messverfahren; } \\
1,5-39 \mu \mathrm{g} / \mathrm{m}^{3}\end{array}$ & & $\begin{array}{l}\text { Keine sign. Assoziation } \\
\text { zwischen Symptomen, } \\
\mathrm{FEV}_{1} \text { und eNO mit FA }\end{array}$ \\
\hline $\begin{array}{l}\text { Wallner et al. } \\
2012 \text { [28] }\end{array}$ & Österreich & 596 & $6-10$ & $\begin{array}{l}\text { Elternfragebogen, } \\
\text { Lungenfunktion }\end{array}$ & $\begin{array}{l}\text { in angrenzenden } \\
\text { Räumen; zweimal; in } \\
\text { jeweils } 2 \text { Klassenräu- } \\
\text { men von } 9 \text { Schulen; } \\
\text { DNPH; HPLC-UV; } \\
\text { Median: } 30 \mu \mathrm{g} / \mathrm{m}^{3} \\
(7-137)\end{array}$ & $\begin{array}{l}\text { Alter, Geschlecht, So- } \\
\text { zialstatus, städtisch/ } \\
\text { ländlich, Anzahl der } \\
\text { Raucher zuhause }\end{array}$ & $\begin{array}{l}\mathrm{MEF}_{50} \text { und } \mathrm{MEF}_{75} \text { sign. } \\
\text { erniedrigt, } \mathrm{FEV}_{1} \text { nicht }\end{array}$ \\
\hline $\begin{array}{l}\text { Yeatts et al. } \\
2012 \text { [29] }\end{array}$ & $\begin{array}{l}\text { Vereinigte } \\
\text { Arabische } \\
\text { Emirate, } \\
2009 / 10\end{array}$ & $\begin{array}{l}1007 \text { ( } 253 \\
\text { Kinder) }\end{array}$ & $6-10$ & $\begin{array}{l}\text { Interviewerfrage- } \\
\text { bogen }\end{array}$ & $\begin{array}{l}\text { Passivsammler, } \\
\text { Kalorimetrie; Median: } \\
<8 \mu \mathrm{g} / \mathrm{m}^{3} \text {; Max: } \\
175 \mu \mathrm{g} / \mathrm{m}^{3}\end{array}$ & $\begin{array}{l}\text { Alter, Geschlecht, Ort, } \\
\text { Passivrauch }\end{array}$ & $\begin{array}{l}\text { Kein sign. Wheezing und } \\
\text { ärztliche Asthmadiagnose } \\
\text { (für die Gesamtgruppe) }\end{array}$ \\
\hline $\begin{array}{l}\text { An- } \\
\text { nesi-Maesa- } \\
\text { no et al. } \\
2012[30]\end{array}$ & Frankreich & 6590 & 10.4 (AM) & $\begin{array}{l}\text { ISAAC Fragebogen, } \\
\text { Pricktest }\end{array}$ & $\begin{array}{l}401 \text { Klassenräumen, } \\
\text { Passivsammler über } 5 \\
\text { Tage, k. A. Mess- } \\
\text { verfahren; Median: } \\
28 \mu \mathrm{g} / \mathrm{m}^{3}(95 . \text { Perzen- } \\
\left.\text { til: } 55 \mu \mathrm{g} / \mathrm{m}^{3}\right)\end{array}$ & $\begin{array}{l}\text { Alter, Geschlecht, Pas- } \\
\text { sivrauch, familiäres } \\
\text { Asthma }\end{array}$ & $\begin{array}{l}\text { Sign. Assoziation mit } \\
\text { Rhinoconjunktivitis im } \\
\text { letzten Jahr }(O R=1,19) \\
\text { aber nicht mit Asthma im } \\
\text { letzten Jahr }\end{array}$ \\
\hline $\begin{array}{l}\text { Dannemiller } \\
\text { et al. } 2013 \\
{[31]}\end{array}$ & $\begin{array}{l}\text { USA, } \\
\text { 2008-10 }\end{array}$ & 46 & 10,5 (AM) & Fragebogen & $\begin{array}{l}30 \text { Min., DNPH, HPLC; } \\
6-165 \mu \mathrm{g} / \mathrm{m}^{3}\end{array}$ & & $\begin{array}{l}\text { Keine sign. Unterschiede } \\
\text { zwischen Asthmasym- } \\
\text { ptomen der Gruppe } \\
\text { mit schwerem Asthma } \\
\text { gegenüber den anderen } \\
\text { Gruppen }\end{array}$ \\
\hline $\begin{array}{l}\text { Madureira } \\
\text { et al. } 2015 \\
{[33]}\end{array}$ & $\begin{array}{l}\text { Portugal, } \\
\text { 2011-13 }\end{array}$ & 1134 & $8-10$ & $\begin{array}{l}\text { Selbstberichteter } \\
\text { Fragebogen, } \\
\text { Lungenfunktions- } \\
\text { prüfung, eNO }\end{array}$ & $\begin{array}{l}\text { Passivsammler, } 5 \\
\text { Tage in } 20 \text { Schulen; } \\
\text { HPLC-UV; Median: } \\
17,5 \mu \mathrm{g} / \mathrm{m}^{3} \text {, Max: } \\
127 \mu \mathrm{g} / \mathrm{m}^{3}\end{array}$ & $\begin{array}{l}\text { Alter, Geschlecht, } \\
\text { mütterliche Bildung, } \\
\text { BMI, Innenraumklima }\end{array}$ & $\begin{array}{l}\text { Sign. Assoziation mit } \\
\text { Wheezing }<12 \mathrm{Mona}- \\
\text { te: } \mathrm{OR}=1,65(95 \% \mathrm{Cl} \text { : } \\
1,02-2,67) \text { nur bei } \mathrm{FA}-\mathrm{Ge}- \\
\text { halten von } 15-20 \mu \mathrm{g} / \mathrm{m}^{3} \text {, } \\
\text { aber nicht bei höheren } \\
\text { Werten; keine Assoziation } \\
\text { mit anderen Symptomen, } \\
\mathrm{FEV}_{1} \text { oder eNO }\end{array}$ \\
\hline \multicolumn{8}{|c|}{ Immunologische Studien } \\
\hline $\begin{array}{l}\text { Wantke et al. } \\
1996 \text { [65] }\end{array}$ & $\begin{array}{l}\text { Österreich, } \\
\text { 1992/3 }\end{array}$ & 62 & $8(\mathrm{AM})$ & $\begin{array}{l}\text { Elternfragebogen, } \\
\text { FA-spezifischer } \\
\text { RAST }\end{array}$ & $\begin{array}{l}5 \text { Tage ungelüftet, } \\
\text { Acetyl-Aceton, Spek- } \\
\text { trometrie; } 30-90 \mu \mathrm{g} / \\
\mathrm{m}^{3}\end{array}$ & & $\begin{array}{l}\text { Konzentrationsabhängige } \\
\text { Korrelation zwischen } \\
\text { Exposition und Sensibili- } \\
\text { sierung (pathologischer } \\
\text { RAST aber nur in } 3 \\
\text { Kindern) }\end{array}$ \\
\hline $\begin{array}{l}\text { Doi et al. } \\
2003 \text { [66] }\end{array}$ & Japan, 1998 & $\begin{array}{l}122 \\
\text { asthmatics } \\
\text { and } 33 \text { Kon- } \\
\text { trollen }\end{array}$ & $3-18$ & $\begin{array}{l}\text { Formaldehyde-spe- } \\
\text { zifische lgE }\end{array}$ & $\begin{array}{l}\text { Keine Innenraumluft- } \\
\text { messung }\end{array}$ & & $\begin{array}{l}\text { Nur in } 2 \text { Asthmatikern } \\
\text { messbare spez. IgE-Kon- } \\
\text { zentrationen }\end{array}$ \\
\hline $\begin{array}{l}\text { Franklin et } \\
\text { al. } 2000 \text { [89] }\end{array}$ & Australien & $\begin{array}{l}224 \text { (Gesun- } \\
\text { de) }\end{array}$ & $6-13$ & $\begin{array}{l}\text { Lungenfunkti- } \\
\text { onsprüfung, eNO, } \\
\text { Picktest }\end{array}$ & $\begin{array}{l}\text { Passivsammler, } \\
\text { Schlaf- und Wohn- } \\
\text { raum über 3-4 Tage, } \\
\text { DNPH, HPLC; Angabe } \\
\text { nur als Gruppierung } \\
\leq 63 \text { oder } \geq 63 \mu \mathrm{g} / \mathrm{m}^{3}\end{array}$ & Alter, Atopiestatus & $\begin{array}{l}\text { Sign. höhere eNO-Ge- } \\
\text { halte bei Kinder aus } \\
\text { Häusern mit } \geq 63 \mu \mathrm{g} / \mathrm{m}^{3} \\
\text { im Vergleich zu } \leq 63 \mu \mathrm{g} / \\
\mathrm{m}^{3} \text { FA; kein Effekt auf } \\
\text { Lungenfunktion }\end{array}$ \\
\hline
\end{tabular}


ben, unter nächtlichem Husten zu leiden oder eine Notfalleinrichtung aufzusuchen (Rückgang zwischen 15 und 20\%). Allerdings war die Schwere des Asthmas, eingeschätzt anhand der Medikamenteneinnahme, in der Interventionsgruppe höher. Nach Auffassung der Autoren stellt die Verringerung der Formaldehyd-Konzentrationen in der Wohnungsluft die plausibelste Erklärung dar. Allerdings wird diese Annahme nicht durch entsprechende Auswertungen (z.B. durch Adjustierung auf die anderen Einflussfaktoren) belegt. Die statistische Power der Studie war für eine belastbare Aussage außerdem unzureichend. Weiterhin ist zu berücksichtigen, dass bekannt ist, dass Interventionsstudien, insbesondere wenn sie mehrere Asthma beeinflussende Faktoren verändert haben, die allgemeine Lebensqualität und die Schwere und Entwicklung von Asthma bei Kindern positiv beeinflussen können [4]. So zeigte sich z. B. auch eine signifikante Reduktion der Asthmamorbidität nach einer Reduktion von Innenraumallergenen [5], von Feinstäuben [6,7] und von Stickoxiden [8].

\section{Kohortenstudien}

Bisher liegen drei Kohortenstudien aus Schweden, Dänemark und Frankreich vor [9-11].

Roda et al. [11] fanden bei 3840 untersuchten Kindern im ersten Lebensjahr eine signifikante Erhöhung des nächtlichen, trockenen Hustens, unabhängig von respiratorischen Erkrankungen, bei einem Anstieg der Formaldehyd-Konzentration um $0,01 \mathrm{mg} / \mathrm{m}^{3}$, allerdings nur bei Kindern, deren Eltern keine Atopie aufwiesen. Bei den anderen Kindern war Husten mit einer Exposition gegenüber biologischen Verunreinigungen der Innenraumluft verknüpft, nicht aber mit einer chemischen Exposition.

Auch Smedje \& Norbäck [9], die 1347 Schüler im Alter von 7-13 Jahren vier Jahre nach der ersten Erhebung nachuntersuchten, fanden bei Kindern ohne eine bekannte Atopie eine signifikante Erhöhung an neuen Asthmadiagnosen. Die Asthmainzidenz nahm pro $0,01 \mathrm{mg}$ Formaldehyd $/ \mathrm{m}^{3}$ Klassenraumluft nicht signifikant zu, aber signifikant für Katzenallergene und Hausstaub.

Bundesgesundheitsbl 2016 ·59:1028-1039 DOI 10.1007/s00103-016-2388-6

(c) Springer-Verlag Berlin Heidelberg 2016

\section{Bekanntmachung des Umweltbundesamtes}

\section{Zur Frage eines Asthma auslösenden bzw. verschlechternden Potenzials von Formaldehyd in der Innenraumluft bei Kindern. Mitteilung des Ausschusses für Innenraumrichtwerte (AIR)}

\section{Zusammenfassung}

Formaldehyd ist ein reaktives, gut wasserlösliches Gas, das eingeatmet insbesondere zu Reizwirkung im Bereich des Nasenrachenraumes führt. Bei chronischer Einwirkung können sehr hohe Formaldehyd-Konzentrationen auch zytotoxische und kanzerogene Wirkungen in diesem Gewebe nach sich ziehen. Ob eingeatmetes Formaldehyd auch in tiefere Teile des Atemtrakts gelangt und insbesondere bei Kindern Asthma auslösen oder verschlimmern kann, wird kontrovers diskutiert. Vor diesem Hintergrund ist es Ziel dieser Übersichtsarbeit den aktuellen Kenntnisstand zu dieser Frage darzustellen und insbesondere die Ergebnisse epidemiologischer Studien kritisch zu diskutieren. Zusammenfassend kann gesagt werden, dass sich auf der Basis der bisher vorliegenden epidemiologischen Studien kein hinreichend belastbarer Zusammenhang zwischen einer Formaldehyd-Exposition in der Innenraumluft und der Entwicklung oder Verschlechterung von Asthma bei Kindern ergibt. Die Aussagekraft einzelner epidemiologischen Studien wird durch eine geringe Stichprobengröße und unplausible Messergebnisse eingeschränkt. Außerdem wurde oft nicht berücksichtigt, dass die Kinder neben Formaldehyd einer Vielzahl weiterer Stoffe bzw. Allergenen ausgesetzt waren. Ergebnisse aus kontrollierten Humanstudien und Tierexperimenten stützen die Auffassung eines fehlenden Zusammenhangs.

Stichworte

Formaldehyd, Innenraumluft, Asthma, Kinder

\section{Association of formaldehyde with the induction or exacerbation of asthma in children.}

\section{Abstract}

Formaldehyde is a highly reactive and water soluble gas and may cause sensory irritations of eyes and nose after inhalation. Moreover, after long-term exposure to high concentrations of formaldehyde cytotoxic and carcinogenic effects were observed.

There is a controversial discussion if formaldehyde is able to reach the deeper parts of the respiratory tract and is associated with the induction or exacerbation of asthma in children. Thus, this review aims in summarizing the current knowledge discussing especially the results from epidemiological studies. On the basis of the current data it can be concluded, that there is no clear association between formaldehyde exposure in the indoor environment and asthma in children. Mainly, the epidemiological studies suffer from small sample sizes, implausible formaldehyde concentrations, and the fact that other substances or factors initiating asthma- and asthma-like complaints were not adequately considered. Results derived from controlled human exposure studies as well as animal experiments support this opinion.

Keywords

Formaldehyde, indoor air, asthma, children
Raaschou-Nielsen et al. [10] untersuchten den Einfluss von Innenraumluftschadstoffen auf die Häufigkeit des Wheezings bei 411 Kindern während der ersten 18 Lebensmonate. Es ließ sich kein Zusammenhang zwischen Verunreinigungen der Innenraumluft (einschließlich Formaldehyd) und täglich von den Eltern notierten Wheezing-Symptomen finden.

In allen drei Studien war die Formaldehyd-Exposition niedrig ([9]: AM $0,008 \mathrm{mg} / \mathrm{m}^{3},[10]:$ AM $0,02 \mathrm{mg} / \mathrm{m}^{3},[11]$ :
Mittel $0,019 \mathrm{mg} / \mathrm{m}^{3}$ ), so dass - soweit überhaupt gesundheitliche Wirkungen gefunden wurden - diese eher anderen Ursachen zuzuordnen sein dürften.

\section{Fall-Kontroll-Studien}

In der Studie von Rumchev et al. [12] wurde Formaldehyd in der Luft des Kinderzimmers und eines weiteren Aufenthaltsraums von 88 asthmatischen Kindern unter 3 Jahren und von 104 altersglei- 
chen Kindern ohne Asthma gemessen. Bei Konzentrationen oberhalb von $0,06 \mathrm{mg}$ Formaldehyd $/ \mathrm{m}^{3}$ ergab sich im Vergleich zu Innenräumen mit $<0,01 \mathrm{mg}$ Formaldehyd $/ \mathrm{m}^{3}$ ein um $39 \%$ erhöhtes Risiko für Asthma. Das Asthmarisiko pro $0,01 \mathrm{mg}$ Formaldehyd $/ \mathrm{m}^{3}$ betrug 1,03 (95\%CI: 1,02-1,04). Deutlich erhöhte Risikowerte wurden auch für ,kindliche Allergie“, „positive Familienanamnese“ und geringere für „Alter“ und „höhere Innenraumtemperaturen“ gefunden. Die Wohnungen der Kinder mit Asthma verfügten häufiger über eine Gasheizung und waren häufiger $(p=0,06)$ frisch renoviert. In einer Nachuntersuchung wurden in den gleichen Wohnungen neben Formaldehyd auch leichtflüchtige Verbindungen, insbesondere Verbrennungsprodukte, in der Luft nachgewiesen [13]. Auch für diese VOCs ergab sich ein Asthmarisiko (OR pro $0,01 \mathrm{mg} / \mathrm{m}^{3}=1.2$ [95\%CI 1,13$1,27])$. Dieses blieb auch dann bestehen, wenn für Formaldehyd adjustiert wurde. Begrenzungen der Aussagekraft der Studie ergeben sich aus der geringen Anzahl an untersuchten Personen, dass Fälle und Kontrollen nicht in der gleichen Institution gewonnen wurden und lediglich Angaben der Eltern zur Diagnose Asthma abgefragt wurden.

Venn et al. [14] untersuchten 193 Kinder mit persistierendem Wheezing und 223 Kontrollen in England. Sie fanden kein erhöhtes Risiko für Wheezing in Relation zur Formaldehyd-Konzentration im Schlafraum der Kinder, aber bei den Fällen war die Formaldehyd-Konzentration mit der Anzahl der nächtlichen Symptome verknüpft. Die Autoren folgern daraus, dass Formaldehyd eher die Schwere der Symptome verstärkt.

Tavernier et al. [15] untersuchten 200 Kinder, davon 105 mit Asthma bzw. eine altersgleichen Kontrollgruppe im Alter von 4-17 Jahren in England. Die Formaldehyd-Konzentrationen in der Wohnungsluft der Kinder mit und ohne Asthma waren nicht unterschiedlich.

Auch in einer Studie mit 73 Kindern in Korea ließen sich keine Unterschiede in den Raumluftgehalten an Formaldehyd in den Wohnungen bei Kindern mit und ohne Asthma finden [16].

In einer französischen Studie wurden 32 Kinder mit Asthma und 31 Kon- trollen in einer Stadt mit 24 bzw. 27 Kindern auf dem Land verglichen [17]. Für die Gesamtgruppe waren lediglich die Toluol- und Acetaldehyd-Konzentrationen in der Wohnungsluft signifikant mit dem Asthmarisiko assoziiert. Nur für die ländliche Untergruppe wurde eine signifikante Assoziation zwischen Asthma und Formaldehyd-Konzentration gefunden $(\mathrm{OR}=10.7 ; 95 \% \mathrm{CI}=1.67-67.6)$.

Hsu et al. [18] untersuchten neben schwerflüchtigen Substanzen auch Formaldehyd in der Luft von Wohnungen von 101 taiwanesischen Kinder im Alter von 3-9 Jahren. Es ergaben sich signifikant niedrigere Gehalte an Formaldehyd in den Wohnungsluft der Untergruppe der Asthmatiker im Vergleich zu den Kontrollen (Median: 0,005 vs. $0,017 \mathrm{mg} / \mathrm{m}^{3}$ ). Allerdings wiesen nur 9 Personen von insgesamt 59 Fällen die Diagnose Asthma auf.

Insgesamt sind die Ergebnisse der aufgeführten Fall-Kontroll-Studien angesichts ihrer kleinen Fallzahlen kritisch zu sehen.

\section{Querschnittsstudien}

Krzyzanowski et al. [19] schlossen 298 Kinder im Alter von 6-15 Jahren, darunter 47 mit der Diagnose Asthma, und 613 Erwachsene in ihre Untersuchung ein. Chronisch respiratorische Symptome waren nicht mit einer inhalativen Formaldehyd-Exposition verknüpft. Es fand sich aber eine höhere Prävalenz an Asthma bei Gehalten über 0,075 $\mathrm{mg}$ Formaldehyd $/ \mathrm{m}^{3}$ in der Küche im Vergleich zu einer niedrigeren Belastung der Innenraumluft. Allerdings bestand diese Beziehung nur, wenn gleichzeitig auch eine Exposition gegenüber Passivrauch vorlag. Außerdem wurde bei den Kindern der exspiratorische Spitzenfluss (PEF) linear mit wachsender Exposition gegenüber Formaldehyd in der Wohnungsluft kleiner. Bei den Erwachsenen wurden keine signifikanten Assoziationen beobachtet.

Smedje et al. [20] untersuchten 762 schwedische Schüler aus 11 Schulen, deren Formaldehydgehalte in der Luft der Klassenräume zwischen $<0,005$ und $0,01 \mathrm{mg} / \mathrm{m}^{3}$ lagen. $6 \%$ der Schüler gaben an, an Asthma zu leiden. Signifikante Assoziationen fanden sich zwischen aktuellem Asthma und höherer relativer Feuch- tigkeit sowie höheren Konzentrationen an Formaldehyd, VOC, Bakterien, Schimmelpilzsporen und Katzenallergenen in der Klassenraumluft.

Garrett et al. [21] untersuchten mittels eines Fragebogens die Häufigkeit von Asthmadiagnosen und respiratorischen Symptomen von 148 Kindern im Alter von 7-14 Jahren, darunter von 53 Kindern mit Asthma. Für drei unterschiedliche Expositionsgruppen (unterteilt nach den Formaldehyd-Konzentrationen in der Innenraumluft der Kinderzimmer) ergaben sich keine signifikanten Unterschiede bezüglich der Asthmahäufigkeit. Wenn allerdings nur die höchsten Werte betrachtet werden, ergab sich ein signifikanter linearer Trend mit mehr asthmatischen Kindern in der höchsten Belastungsgruppe $\left(>0,05 \mathrm{mg} / \mathrm{m}^{3}\right)$. In der logistischen Regression unterschied sich das Asthmarisiko nach Adjustierung auf elterliches Asthma/Allergie nicht mehr signifikant. Es finden sich keine Angaben zur Passivrauchbelastung der untersuchten Gruppen und keine Berücksichtigung der sozialen Lage der Studienteilnehmer. Für das gleiche Studienkollektiv wird in einer anderen Veröffentlichung beschrieben, dass bestimmte Schimmelpilzgruppen, nicht aber der Gesamtgehalt an aerogenen Sporen, ein Risikofaktor für die Asthma-, Allergieentstehung und respiratorische Symptome darstellen [22]. Diese Risikofaktoren wurden bei der zweiten Studie jedoch nicht als Störgrößen berücksichtigt. Außerdem liegen die zwei ländlichen Studienorte in unmittelbarer Nähe zu Braunkohleabbaugebieten mit mehreren Kraftwerken, die als Expositionsquelle unberücksichtigt bleiben.

Jaakola et al. [23] führten eine Querschnittsstudie an 5951 russischen Schulkindern durch. Es ergab sich eine signifikante Assoziation zwischen der Verwendung von Spanplatten in der Wohnung und aktuellem Wheezing (jedoch nur, wenn der Einbau länger als 12 Monate zurücklag), aber nicht mit aktuellem Asthma. Formaldehyd in der Innenraumluft wurde in dieser Studie nicht gemessen.

Mi et al. [24] untersuchten 1414 Schulkinder aus 10 Schulen in Shanghai. Während für einige Luftschadstoffe wie $\mathrm{CO}_{2}$ und $\mathrm{NO}_{2}$ Assoziation gefunden wurde, ergab sich für Formaldehyd in der multiplen lo- 
gistischen Regression kein Zusammenhang zwischen Formaldehyd in der Schulklassenluft und asthmabezogenen Symptomen (z.B. Wheezing und Kurzatmigkeit) oder dem Vorliegen eines aktuellen Asthmas.

Die Studie von Zhao et al. [25] umfasste 1480 Schulkinder aus 10 chinesischen Schulen im Alter von 11-15 Jahren. Die Formaldehyd-Konzentration betrug $0,001-0,005 \mathrm{mg} / \mathrm{m}^{3}$. Bei Anwendung eines hierarchischen multiplen logistischen Regressionsmodells wurde keine signifikante Assoziation zwischen der Formaldehyd-Konzentration in der Luft der Klassenräume mit Wheezing und Kurzatmigkeit bei den Schulkindern gefunden.

Kim et al. [26] schlossen 1028 Kinder aus 12 koreanischen Schulen in ihre Studie ein. Bei Formaldehydgehalten zwischen 0,016 und $0,047 \mathrm{mg} / \mathrm{m}^{3}$ ergaben sich keine signifikanten Assoziationen zwischen der Formaldehydexposition und Wheezing, einem vom Arzt diagnostizierten Asthma oder aktuellem Asthma.

Martins et al. [27] untersuchten die Innenraumluft in Schulen und Wohnungen von 51 Kinder aus Portugal. Neben einem Elternfragebogen wurde bei allen Kindern eine Spirometrie durchgeführt und eNO in der Ausatemluft gemessen. Die Formaldehydgehalte in der Innenraumluft waren nicht signifikant mit Lungenfunktionsparametern, dem eNO, Wheezing, der Notwendigkeit einer Notfallmedikation oder dem Aufsuchen einer Notfalleinrichtung assoziiert.

Wallner et al. [28] untersuchten 596 Kinder im Alter von 6-10 Jahren aus 9 österreichischen Schulen. In der Lungenfunktion wurde eine signifikante negative Assoziation von Formaldehyd in der Innenraumluft (Median 0,03 $\mathrm{mg} / \mathrm{m}^{3}$ ) zum $\mathrm{MEF}_{50}$ und $\mathrm{MEF}_{75}$, die auf eine Beeinflussungen der mittleren und großen Atemwege schließen lässt, gefunden. $\mathrm{FEV}_{1}$ war nicht signifikant von den Formaldehydkonzentrationen beeinflusst. Angaben zu Ergebnissen des Elternfragebogens geben die Autoren nicht.

Yeatts et al. [29] schlossen in ihrer Studie 253 Kinder aus den Arabischen Emiraten ein. Für einige respiratorische Symptome (Kurzatmigkeit, Husten) wurden positive Assoziationen mit Formaldehyd in der Innenraumluft (Median 0,008 mg/ $\mathrm{m}^{3}$ ) gefunden, nicht jedoch für Wheezing, vom Arzt diagnostiziertes Asthma und nächtlicher Husten.

Annesi-Maesano et al. [30] führten Untersuchungen an 6590 französische Schulkindern aus 108 Schulen durch. Während sich für eine Rhinokonjunktivitis im zurückliegenden Jahr eine signifikante Assoziation mit der Höhe der Formaldehyd-Konzentrationen in der Klassenraumluft (Median 0,028 mg/m $\mathrm{m}^{3}$ ) ergab, ließ sich diese Beziehung für Asthma nicht finden.

Dannemiller et al. [31] erhoben bei 46 Kindern mit Asthma Daten zur Häufigkeit von Symptomen, zum Medikamentenverbrauch und zu Fehlzeiten in der Schule. 6 Kinder wurden als besonders schwer von Asthma betroffen eingestuft. Die mittlere Formaldehyd-Konzentration in der Wohnungsluft dieser Kinder betrug 0,068 mg/ $\mathrm{m}^{3}$, während sie in der Wohnungsluft weniger betroffener Kinder bei $0,034 \mathrm{mg} / \mathrm{m}^{3}$ lag. Es ergaben sich keine signifikanten Unterschiede zwischen asthmabezogenen Symptomen und Formaldehyd, wenn die schwerer betroffene Gruppe mit den anderen Gruppen verglichen wurde.

Zhai et al. [32] fanden bei der Untersuchung der Luft von 82 Kinderzimmern in 20 Fällen Konzentrationen über $0,08 \mathrm{mg}$ Formaldehyd $/ \mathrm{m}^{3}$. Auf Befragung gaben die Eltern an, von den 20 Kindern in diesen Wohnungen hätten $40 \%$ Asthma, während von den 62 Kindern mit niedrigeren Formaldehyd-Konzentrationen nur $3 \%$ Asthma hätten.

Madureira et al. [33] untersuchten 1134 portugiesische Kinder aus 20 Schulen. Neben Innenraumluftmessungen wurde auch einmal eine Lungenfunktion durchgeführt und eNO in der Ausatemluft bestimmt. Lediglich für das Wheezing innerhalb der letzten 12 Monate fand sich eine signifikante Assoziation für die Expositionsgruppe zwischen 0,015 und $0,02 \mathrm{mg}$ Formaldehyd $/ \mathrm{m}^{3}$, aber nicht für die höher belastete Gruppe. Für Wheezing in den letzten 30 Tagen, Hustenepisoden, Lungenfunktionsparametern oder eNO ergaben sich keine signifikanten Assoziationen.

Im Unterschied $\mathrm{zu}$ den Fall-Kontroll-Studien wurden in einigen Querschnittsstudien größere Fallzahlen erreicht. Auch hierbei ergab sich kein belastbarer Zusammenhang zwischen einer
Exposition gegenüber Formaldehyd in der Innenraumluft und Asthma.

\section{Metaanalysen}

Bisher sind zwei Metaanalysen veröffentlicht worden:

McGwin et al. [34] berücksichtigten 7 Studien an 5930 Kindern, von denen 364 die Diagnose Asthma hatten $[9,12,19,20,21,24,25]$. Hierbei wurden zwei statistische Verfahren angewendet. In einem Modell ergab sich eine $3 \%$ ige Zunahme des Asthmarisikos pro 0,01 mg Formaldehyd $/ \mathrm{m}^{3}$ Innenraumluft (95\% CI 1.02-1.04), im anderen Modell eine $17 \%$ ige Zunahme (95\% CI 1.01-1.36). Die $3 \%$ ige Risikoerhöhung beruhte zu 99\% auf der Studie von Rumchev et al. [12], bei der $17 \%$ igen Risikoerhöhung trug diese nur zu 35\%, die Garrett et al. [21] Studie $\mathrm{zu} 22 \%$ und die anderen mit jeweils bis zu $15 \%$ zur Risikoerhöhung bei.

Yao et al. [35] wählten vier chinesische und zwei europäische [17,36] Fall-Kontrollstudien mit insgesamt 362 Kindern mit Asthma und 356 Kontrollen ohne Asthma aus. In den Fallgruppen betrug die mittlere Formaldehyd-Konzentration 0,03-0,13 mg/m $\mathrm{m}^{3}$, in den Kontrollgruppen 0,02-0,085 mg/m $\mathrm{m}^{3}$. Nach Ansicht der Autoren deutet der gewichtete Unterschied der Formaldehyd-Konzentration zwischen den Fall- und Kontrollgruppen von im Mittel 0,02 mg/ $\mathrm{m}^{3}$ auf Formaldehyd als einen Risikofaktor für die Entstehung von Asthma hin. Die vier chinesischen Studien ließen sich nicht über die üblichen Suchwege finden und können somit nicht im Original beurteilt werden. In einer verwendeten Veröffentlichung wurden nicht Kinder, sondern Erwachsene im Alter von 20-45 Jahren untersucht [36].

\section{Sonstige Studien zum Zusammenhang inhalative Formaldehyd-Exposition und Asthma}

\section{Tierexperimentelle Studien}

Bei den tierexperimentellen Untersuchungen zur Ermittlung eines Zusammenhangs zwischen Formaldehyd-Exposition und der Entstehung bzw. Verschlechterung von Asthma standen Entzündungs- 
reaktionen und die Auslösung von Überempfindlichkeiten im Atemtrakt nach inhalativer Exposition gegenüber Formaldehyd im Vordergrund.

Bisher sind 7 Studien an Mäusen [3743], eine an Ratten [44] und zwei an Meerschweinchen $[45,46]$ durchgeführt worden. In ihnen wurden Tiere über 6-24 Stunden pro Tag über einen Zeitraumes von 5 bis 84 Tagen gegenüber Formaldehyd in einem relevanten Dosisbereich exponiert. Die Effekte wurden in der Regel für Formaldehyd allein und nach Sensibilisierung mit Ovalbumin (OVA) ermittelt. Signifikante Effekte (z.B. erhöhte Anzahl eosinofiler Zellen in der Bronchiallavage, Zunahme von proinflammatorischen $\mathrm{Zy}$ tokinen und Chemokinen, erhöhte Antikörperproduktion) wurden bei Mäusen und Ratten bei einer Exposition gegenüber 1-3 mg Formaldehyd $/ \mathrm{m}^{3}$ und dort auch nur in den mit OVA behandelten Gruppen beobachtet. Bei Meerschweinchen wurden in der Studie von Riedel et al. [45] gleichfalls keine Entzündungszeichen bei den Tieren gesehen, die 0,16 oder $0,31 \mathrm{mg}$ Formaldehyd $/ \mathrm{m}^{3}$ allein ausgesetzt waren, wohl aber bei Tieren der Dosisstufe von $0,31 \mathrm{mg} / \mathrm{m}^{3}$ nach OVA-Sensibilisierung. Swiecichowski et al. [46] beobachteten nach 8-stündiger Exposition von Meerschweinchen eine signifikante Zunahme des Atemwegswiderstandes bei 0,38 mg/ $\mathrm{m}^{3}$, nicht aber bei $0,12 \mathrm{mg} / \mathrm{m}^{3}$. Bei einer 2-stündigen Exposition traten diese Effekte erst ab einer sehr hohen Konzentration von 11,3 mg Formaldehyd $/ \mathrm{m}^{3}$ auf.

Insgesamt deuten die tierexperimentellen Untersuchungen darauf hin, dass Wirkungen teilweise erst bei sehr hohen Formaldehyd-Konzentrationen auftraten und -wenn überhaupt- Formaldehyd höchstens einen adjuvanten Effekt hinsichtlich der Auslösung von Asthma haben könnte. Aufgrund von Speziesunterschieden in der immunologischen Reaktion, insbesondere im Hinblick auf den OVA-Test, wird eine direkte Übertragung dieser tierexperimentellen Ergebnisse auf den Menschen jedoch als kritisch angesehen.

\section{Inhalative Expositionsstudien an Erwachsenen}

Nordman et al. [47] untersuchten 230 über längere Zeit Formaldehyd-exponierte Be- schäftigte mit asthmaähnlichen Symptomen. In der bronchialen Provokation mit Formaldehyd zeigten 12 Arbeiter eine Abnahme der Lungenfunktionsparameter 15 Minuten bis 24 Stunden nach einer $30 \mathrm{mi}-$ nütigen Formaldehyd-Konzentration von $2,5 \mathrm{mg} / \mathrm{m}^{3}$ (bei einem Beschäftigten bereits bei $\left.1,2 \mathrm{mg} / \mathrm{m}^{3}\right)$.

Sheppard et al. [48] exponierten 7 Asthmatiker über 10 Minuten gegenüber $1,24 \mathrm{mg}$ Formaldehyd $/ \mathrm{m}^{3}$ und fanden sowohl in Ruhe als auch bei Anstrengung keine Zeichen einer Bronchokonstriktion.

Frigas et al. [49] untersuchten 13 Asthmatiker, die 20 Minuten gegenüber $0,12,1,2$ oder $3,1 \mathrm{mg}$ Formaldehyd $/ \mathrm{m}^{3}$ exponiert waren, und fanden keine Änderungen in der Lungenfunktion im Vergleich zur Inhalation von nicht Formaldehyd-belasteter Luft.

Sauder et al. [50] exponierten 9 Asthmatiker über 3 Stunden mit 3,7 mg Formaldehyd $/ \mathrm{m}^{3}$ und fanden keine signifikanten Änderungen in der Lungenfunktion, wohl aber irritative Effekte im Bereich des Nasen-Rachenraumes und der Augen.

Green et al. [51] exponierten $22 \mathrm{Ge}$ sunde und 16 Asthmatiker über 1 Stunde gegenüber $3,7 \mathrm{mg}$ Formaldehyd $/ \mathrm{m}^{3}$. Bei körperlicher Anstrengung wurde bei den Lungengesunden, nicht aber den Asthmatikern eine geringe Erniedrigung der Lungenfunktionsparameter beobachtet (mittlere Abnahme von $\mathrm{FEV}_{1}$ um 3,8\%). $13 \%$ der Versuchspersonen zeigten bei dieser Formaldehyd-Konzentration einen klinisch relevanten $\mathrm{FEV}_{1}$-Abfall von $>10 \%$.

Uba et al. [52] untersuchten in einer prospektiven Studie unter anderem die Lungenfunktion und asthmabezogene respiratorische Symptome bei 103 Medizinstudenten über 7 Monate. Weder Lungengesunde noch Asthmatiker zeigten signifikante Assoziationen bezüglich der Formaldehyd-Exposition (im Mittel unter $1 \mathrm{ml} / \mathrm{m}^{3}$ mit Spitzen unter $5 \mathrm{ml} / \mathrm{m}^{3}$ ). Es wurde signifikant mehr Reizungen an Auge und im oberen Atemtrakt angegeben.

Harving et al. [53] untersuchten 15 behandlungspflichtige Asthmatiker, die über 90 Minuten Konzentrationen von 0,008 (Kontrolle), 0,12 und 0,85 mg Formal$\mathrm{dehyd} / \mathrm{m}^{3}$ ausgesetzt waren. Es zeigten sich keine signifikanten Veränderungen in der Lungenfunktion, keine Spätreaktion 14-16
Stunden nach dem Experiment und kein Hinweis auf eine veränderte bronchiale Reaktion nach Provokation mit Histamin.

Krakowiak et al. [54] exponierten 10 Gesunde und 10 Probanden mit Rhinitis und Asthma einmalig über einen Zeitraum von 2 Stunden gegenüber $0,5 \mathrm{mg}$ Formaldehyd $/ \mathrm{m}^{3}$. Auch nach Provokation ergaben sich keine Zeichen einer allergischen Reaktion in den oberen und tiefen Atemwegen und keine Unterschiede zwischen Gesunden und Kranken.

Casset et al. [55] fanden in einer verblindeten Studie mit 19 Asthmatikern, die eine halbe Stunde lang 0,1 mg Formaldehyd $/ \mathrm{m}^{3}$ oder unbelasteteter Luft ausgesetzt waren, keine Änderungen in der Lungenfunktion, der Eosinophilen im Blut und Sputum und der induzierten Symptome. Allerdings beschreiben die Autoren eine verstärke Sofort- und Spätwirkung nach Inhalation von Milbenallergen bei vorangegangener Formaldehyd-Exposition im Vergleich zur Kontrolle nach Inhalation von Milbenallergen (FEV ${ }_{1}$ um $15 \%$ bzw. $11 \%$ reduziert).

Ezratty et al. [56] exponierten in einer verblindeten Cross-over-Studie 12 Pollenallergiker mit intermitierendem Asthma über eine Stunde mit jeweils 0,5 mg Formaldehyd $/ \mathrm{m}^{3}$. Es ergaben sich keine signifikanten Effekte auf die Lungenfunktion und keine Entzündungszeichen (z. B. Zellzahlen, Chemokine im Sputum) in den Atemwegen.

Insgesamt ließen sich in neun der zehn Studien selbst bei hohen Formaldehyd-Konzentrationen $>1 \mathrm{mg} / \mathrm{m}^{3}$ kaum Hinweise auf eine Verschlechterung von Lungenfunktionsparametern oder Entzündungszeichen weder bei Gesunden noch bei Asthmatikern finden. Lediglich in einer älteren Studie wurde ein Effekt auf die Lungenfunktion bei langfristig exponierten Arbeitern gefunden, die in einem Provokationstest hohen Formaldehyd-Konzentrationen von 2,5 mg/ $\mathrm{m}^{3}$ ausgesetzt waren.

\section{Studien zu spezifischem IgE nach Formaldehyd-Exposition}

\section{Studien an Erwachsenen}

Wilhelmsson und Holmström [57] untersuchten 30 Arbeiter mit einer langjähri- 
gen mittleren Exposition um $1 \mathrm{mg}$ Formaldehyd $/ \mathrm{m}^{3}$. Bei 2 Beschäftigten war Formaldehyd-spezifisches IgE im Serum nachweisbar. Beide zeigten nasale Symptome, asthmatische Beschwerden wurden nicht mitgeteilt.

Kramps et al. [58] untersuchten 86 Serumproben auf spezifisches IgE von Personen, die am Arbeitsplatz oder während medizinischer Behandlungen gegenüber Formaldehyd exponiert waren. Nur in einem Fall wurde spezifisches IgE gefunden, wobei der Arbeiter keine typischen Symptome angab.

Grammer et al. [59] fanden bei 37 Formaldehyd-exponierten Arbeitern keine spezifischen IgE- oder IgG-Antikörper. Daneben berichtet die Arbeitsgruppe von einem Arbeiter, bei dem spezifisches IgE gefunden wurden, bei dem aber selbst bei einer 20minütigen Exposition gegenüber $6,2 \mathrm{mg}$ Formaldehyd $/ \mathrm{m}^{3}$ keine respiratorischen Effekte ausgelöst werden konnten [60].

Dykewicz et al. [61] konnten bei 3 von 55 Personen Formaldehyd-spezifische IgE-Antikörper nachweisen. Lediglich eine dieser Personen schilderte respiratorische Symptome während der Arbeitszeit in einem Histologielabor. Bei keiner Person konnten bei einmaliger Inhalation von $2,5 \mathrm{mg}$ Formaldehyd $/ \mathrm{m}^{3}$ Symptome ausgelöst werden.

Liden et al. [62] fanden bei 23 Patienten im Alter von 21-74 Jahren nur 2 mit Formaldehyd-spezifischen IgE. Beide zeigten keine Symptome einer Atopie.

Wandtke et al. [63] untersuchten 45 Medizinstudenten im Alter von 1833 Jahren, die im Rahmen eines Anatomiekurses Phenol und Formaldehyd ausgesetzt waren, auf spezifisches IgE und gesundheitliche Beschwerden. Die Formaldehyd-Konzentration in den Arbeitsräumen war eher niedrig $(0,03-0,27 \mathrm{mg} /$ $\mathrm{m}^{3}$; im Mittel $\left.0,16 \mathrm{mg} / \mathrm{m}^{3}\right)$. Nach einer einmonatigen Exposition (3 Stunden täglich) konnten keine IgE bei den Studenten nachgewiesen werden. In einer Nachuntersuchung von 27 Studenten des vorgenannten Kollektivs während eines zweiten Kurses wurden am Anfang keine Formaldehyd-spezifischen IgE oder IgG gefunden [64]. Am Ende ließ sich bei 4 Studenten spezifisches IgE - aber nicht IgG - nachweisen, wobei keine Korrelation mit den erhobenen Symptomen beobachtet wurde. Die mittlere Belastungssituation lag in dieser Gruppe bei 4,65 mg Phe$\mathrm{nol} / \mathrm{m}^{3}$ und $0,27 \mathrm{mg}$ Formaldehyd $/ \mathrm{m}^{3}$.

\section{Studien an Kindern}

Wantke et al. [65] untersuchten 62 Kinder (mittleres Alter 8 Jahre) aus drei Schulen mit Formaldehyd-Konzentration zwischen $0,05-0,09 \mathrm{mg} / \mathrm{m}^{3}$. Bei 3 Schülern wurde deutlich erhöhte spezifische IgE gefunden, wobei 2 Kinder aus dem am höchsten belasteten Klassenraum stammten. Bei weiteren 21 wurden erhöhte IgE gefunden. Bei den anderen Kindern sowie 19 Kontrollen (Formaldehyd-Exposition nicht angegeben) waren keine IgE nachweisbar. Die IgE-Werte korrelierten nicht mit der Anzahl und Schwere der Symptome. Angaben zur Exposition der Kinder gegenüber Formaldehyd in anderen relevanten Aufenthaltsräumen (z.B. Wohnung) fehlen.

Doi et al. [66] untersuchten Formaldehyd-spezifisches IgE in 122 Kindern mit Asthma und 33 nichtallergischen Kontrollen (3-18 Jahre). Nur bei 2 Asthmatikern konnte Formaldehyd-spezifisches IgE in niedrigen Konzentrationen ( 0,42 und 0,46 $\mathrm{UA} / \mathrm{ml}$ ) gefunden werden. Angaben zur Expositionssituation der Kinder fehlen.

Zusammenfassend zeigen die Untersuchungen keinen Zusammenhang zwischen einer Exposition gegenüber Formaldehyd in der Innenraumluft und einer spezifischen immunologischen Reaktion. Bei Erwachsenen traten sehr selten erhöhte IgE-Antikörper und -wenn überhaupt- nur bei hohen Luftkonzentrationen an Formaldehyd auf, diese waren aber nicht mit respiratorischen Symptomen verknüpft waren. Eine dermale Exposition gegenüber Formaldehyd lag entweder vor oder kann nicht sicher ausgeschlossen werden. Bei Erwachsenen stellt Formaldehyd in der Luft von Arbeitsplätzen daher keine wesentliche Ursache von immunologisch beeinflussten Atemwegsreaktionen dar. Gleiches scheint auch für Kinder zuzutreffen. Allerdings lässt die sehr begrenzte Datenlage keine valide Einschätzung zu.

\section{Bewertung der Studien zu Asthma und Formaldehyd in der Innenraumluft}

\section{Bewertung in wissenschaftlichen Übersichtsarbeiten}

Zur gesundheitlichen Wirkung eingeatmeten Formaldehyds sind zahlreiche zusammenfassende Darstellungen erschienen. In aktuellen Übersichtsarbeiten sehen die Autoren keine belastbaren Anhaltspunkte für eine Auslösung oder Verschlechterung von Asthma durch Formaldehyd in der Innenraumluft [67-73].

\section{Bewertung von internationalen Organisationen}

Nach Auffassung der Weltgesundheitsorganisation wurden bislang weder in epidemiologischen Studien noch in Expositionskammeruntersuchungen konsistente Ursache-Wirkungs- oder Dosis-Wirkungsbeziehungen zwischen einer Exposition unterhalb von $1 \mathrm{mg}$ Formaldehyd $/ \mathrm{m}^{3}$ in der Innenraumluft und nachweisbaren Lungenfunktionsänderungen gefunden [1]. Soweit in epidemiologischen Untersuchungen in Wohnungen oder Schulen Effekte beobachtet wurden, konnte die WHO eine Entstehung bzw. Verstärkung von Asthma bzw. asthmabezogenen Symptomen angesichts weiterer Einflussfaktoren nicht eindeutig dem Formaldehyd zuordnen.

Die US-amerikanische Umweltschutzbehörde hat im Rahmen der Überarbeitung der inhalativen Reference concentration (RfC) für Formaldehyd im Integrated Risk Information System (IRIS) 2010 einen umfangreichen Berichtsentwurf publiziert [74]. Nach Darstellung der einzelnen Studienergebnisse kommt sie in der Zusammenfassung zu dem Schluss, dass u. a. eine Lungenfunktionseinschränkung oder eine Sensibilisierung (Atopie und Asthma) einen möglichen Endpunkt für die Ableitung einer inhalativen RfC für Formaldehyd darstellen und die Studien von Rumchev et al. [12], Garrett et al. [21] und Krzyzanowski et al. [19] hierfür herangezogen werden könnten. Eine kritische Diskussion dieser Studien und ihrer Einschränkungen (z. B. hinsichtlich von Koexpositionen) erfolgte nicht. Eine 
abschließende Entscheidung der US-EPA, ob dieser Endpunkt für eine Ableitung einer RfC geeignet sein könnte, ist bislang (Stand 20.2.2016) nicht erfolgt.

Die US-amerikanische Agency for Toxic Substances and Disease Registry (ATSDR) hat 1999 eine umfangreiche Übersichtsarbeit zu Formaldehyd in der Innenraumluft veröffentlicht und sah bei dem damaligen Stand der wissenschaftlichen Kenntnisse die Ergebnisse bezüglich Asthma kritisch und zudem keine Möglichkeit, die Frage der besonderen Empfindlichkeit von Kindern zu klären [75]. In einem Addendum von 2010 kommt die ATSDR zu dem Schluss, dass einige Studien Hinweise darauf geben, dass Formaldehyd in der Luft von Innenräumen Kleinkinder für Asthma und Allergien prädisponieren könnte, allerdings sehen sie keinen verlässlichen Dosis-Wirkungs-Zusammenhang [76].

Das Committee on the Assessment of Asthma and Indoor Air des Institute of Medicine kommt in seiner Zusammenfassung im Jahr 2000 zu dem Ergebnis, dass für Formaldehyd nur eine begrenzte oder widersprüchliche Evidenz bezüglich einer Assoziation mit respiratorischen Symptomen vorliegt und die Evidenz bezüglich des Asthmaentwicklung inadäquat oder begrenzt ist [77]. In einem Update wurde diese Einschätzung bestätigt [78]. Ausreichende Hinweise für einen ursächlichen Zusammenhang zu bestimmten Substanzen in der Innenraumluft sehen die Autoren lediglich bei Kindern, die gegen Feuchteschäden bzw. damit verbundenen Agenzien, Endotoxinen sowie Passivrauch exponiert sind.

\section{Diagnostische Schwierigkeiten mit Asthma bei Kindern in epidemiologischen Studien}

Unter Asthma versteht man eine komplexe chronisch-entzündliche Erkrankung der Atemwege. Charakteristisch für Asthma ist eine reversible Verengung der Atemwege. Die ärztliche Diagnose eines Asthmas beruht neben der Anamnese, der körperlichen Untersuchung und eines Lungenfunktionstestes auf der Bewertung des Verlaufs der Reversibilität der Atemwegsverengung durch wiederholte Untersuchungen sowie dem Ausschluss anderer Erkrankungen mit vergleichbarer
Symptomatik [68]. Angesichts der größeren Fallzahlen wird das Kriterium Reversibilität in epidemiologischen Studien in der Regel nicht geprüft, sondern sich auf Anamnese, körperliche Untersuchung und Lungenfunktionstest beschränkt. In vielen Studien beruht die Diagnose „Asthma" auf einer Befragung des Kindes oder der Eltern, eine ärztliche Diagnostik wurde nicht durchgeführt. Besonders kritisch ist die Diagnose Asthma bei Kindern unter 6-8 Jahren zu hinterfragen, da in dieser Altersgruppe die Reversibilität besonders schwer einzuschätzen ist. Gleiches trifft auch auf das Wheezing im Kindesalter zu. Bei ca. $50 \%$ der Kinder treten bis zum 6. Lebensjahr mindestens einmal Atemgeräusche auf, die jedoch oft wieder verschwinden und pathophysiologisch nicht mit den Veränderungen beim Asthma vergleichbar sind [79].

In der Studie von Rumchev et al. [12] wurde versucht, Asthma bei Kindern unter 3 Jahren lediglich durch einen Elternfragebogen zu ermitteln. In der Metaanalyse von McGwin et al. [34] dominiert gerade diese Studie das Ergebnis zu 35-99\%. Damit ist diese Metaanalyse mit erheblichen Unsicherheiten behaftet. Auch in den Studien von Krzyzanowski et al. [19] und Garrett et al. [21], in denen ältere Kinder untersucht wurden, ist die Diagnose „Asthma“ allein aufgrund der elterlichen Angaben mit großen Unsicherheiten behaftet.

\section{Andere potenzielle \\ Expositionsfaktoren für kindliches Asthma}

In den letzten Jahrzehnten wurden neben den genetischen und sozialen Faktoren [80] auch zahlreiche weitere Verunreinigungen in der Innenraumluft neben Formaldehyd identifiziert und ein möglicher Zusammenhang mit der Entstehung und Verschlechterung von Asthma bei Kindern untersucht [7,68,71-73,8186]. Hierbei handelt es sich z. B. um Passivrauch, Stickoxide, Feinstäube, der Betrieb von Gasherden, die Verwendung von Reinigungsmitteln, die Renovierung von Kinderzimmern und anderen Aufenthaltsräumen von Kindern, die Verwendung von Kinderspielzeug und anderen Gebrauchsgegenständen aus PVC mit einem hohen Anteil von Weichmachern, die Haltung von Haustieren insbesondere von Katzen, das Vorkommen von Hausstaubmilben und Schimmelbefall.

\section{Zusammenfassende Bewertung}

Formaldehyd zählt zu den reaktiven, gut wasserlöslichen Substanzen. In neueren, auf anthropometrischen Daten basierenden Expositionsmodellen des Atemtraktes konnte gezeigt werden, dass gasförmige Substanzen mit diesen Eigenschaften praktisch vollständig im extratrachealen Bereich (Nasenrachenraum), in dem die Reizwirkung von Formaldehyd auftritt, resorbiert werden und keine wesentlichen toxikokinetischen Unterschiede zwischen Kindern und Erwachsenen beobachtet wurden $[87,88]$. Ob toxikodynamische Unterschiede $\mathrm{zu}$ berücksichtigen sind, z. B. eine höhere Empfindlichkeit des Nasenepithels von Kindern, ist unbekannt.

Bei Erwachsenen zeigten sich sowohl unter kontrollierten Expositionsbedingungen als auch in epidemiologischen Studien an Arbeitsplätzen bei Konzentrationen bis $1 \mathrm{mg}$ Formaldehyd $/ \mathrm{m}^{3} \mathrm{kei}$ ne Veränderungen der Lungenfunktion, auch nicht bei Asthmatikern. Studien mit kontrollierten Expositionsbedingungen gegenüber Formaldehyd an Kindern liegen nicht vor. In epidemiologischen Studien an Kindern ergab sich meist kein hinreichend belastbarer Zusammenhang zwischen einer Formaldehyd-Exposition und der Entwicklung oder Verschlechterung von Asthma. Soweit ein solcher $\mathrm{Zu}$ sammenhang gefunden wurden, konnte er nicht eindeutig Formaldehyd zugeordnet werden, da die Kinder neben Formaldehyd einer Vielzahl weiterer Stoffe bzw. Allergenen ausgesetzt waren. Auch wird die Aussagekraft einzelner epidemiologischer Studien durch eine geringe Stichprobengröße zusätzlich eingeschränkt. Auch die Ergebnisse der tierexperimentellen Untersuchungen lassen einen Zusammenhang bei der um Größenordnungen niedrigeren Expositionssituation von Kindern in epidemiologischen Studien wenig wahrscheinlich erscheinen.

Vor diesem Hintergrund ergeben sich auch aus der Berücksichtigung der aktuellen Literatur keine neuen Anhaltspunkte gegenüber den bisher veröffentlichten Einschätzungen. Es lassen sich keine Bele- 
ge für einen belastbaren Zusammenhang zwischen einer Formaldehyd-Exposition in der Innenraumluft und einer Entwicklung oder Verschlechterung von kindlichem Asthma finden.

Danksagung. Wir bedanken uns bei Herrn Joachim Heinrich für die kritische inhaltliche Durchsicht des Manuskriptes.

Anmerkung. Der Text dieser Mitteilung wurde von Prof. Dr. Hermann Fromme und Dr. Helmut Sagunski erstellt und vom Ausschuss für Innenraumrichtwerte im Mai 2016 verabschiedet. Die Literaturrecherche wurde im März 2016 abgeschlossen.

\section{Literatur}

1. WHO (World Health Organization) (2010) WHO guidelines for indoor air quality: selected pollutants. World Health Organization, Regional Office for Europe, Copenhagen. www.euro.who. int/_data/assets/pdf_file/0009/128169/e94535. pdf. Zugegriffen: 1. März 2016

2. Schroeter JD, Campbell J, Kimbell JS, Conolly RB, Clewell HJ, Andersen ME (2014) Effects of endogenous formaldehyde in nasal tissues on inhaled formaldehyde dosimetry predictions in the rat, monkey, and human nasal passages. Toxicol Sci 138:412-424

3. Lajoie $P$, Aubin D, Gingras V, Daigneault $P$, Ducharme F, Gauvin FD, Fugler D, Leclerc JM, Won $D$, Won D, Courteau M, Gingras S, Héroux MĖ, Yang W, Schleibinger $H$ (2015) The IVAIRE project - A randomized controlled study of the impact of ventilation on indoor air quality and the respiratory symptoms of asthmatic children in single family homes. Indoor Air 25:582-597

4. Crocker DD, Kinyota S, Dumitru GG, Ligon CB, Herman EJ, Ferdinands JM, Hopkins DP, Lawrence BM, Sipe TA (2011) Effectiveness of home-based, multi-trigger, multicomponent interventions with an environmental focus for reducing asthma morbidity: a community guide systematic review. Am J Prev Med 41(2):S5-S32

5. Morgan WJ, Crain EF, Gruchalla RS, O'Connor GT, Kattan M, Evans R 3rd, Stout J, Malindzak G, Smartt E, Plaut M, Walter M, Vaughn B, Mitchell $\mathrm{H}$, Inner-City Asthma Study Group (2004) Results of a home-based environmental intervention among urban children with asthma. N Engl J Med 351:1068-1080

6. Eggleston PA, Butz A, Rand C, Curtin-Brosnan J, Kanchanaraksa S, Swartz L, Breysse P, Buckley T, Diette G, Merriman B, Krishnan JA (2005) Home environmental intervention in inner-city asthma: a randomized controlled clinical trial. Ann Allergy Asthma Immunol 95:518-524

7. Breysse PN, Diette GB, Matsui EC, Butz AM, Hansel NN, McCormack MC (2010) Indoor air pollution and asthma in children. Proc Am Thorac Soc 7(2):102-106

8. Pilotto LS, Nitschke M, Smith BJ, Pisaniello D, Ruffin RE, McElroy HJ, Martin J, Hiller JE (2004) Randomized controlled trial of unflued gas heater replacement on respiratory health of asthmatic schoolchildren. Int J Epidemiol 33:208-214

9. Smedje G, Norbäck D (2001) Incidence of asthma diagnosis and self-reported allergy in relation to the school environment - a four-year follow-up study in schoolchildren. Int J Tuberc Lung Dis 5:1059-1066

10. Nielsen, Hermansen MN, Loland L, Buchvald F, Pipper CB, Sørensen M, Loft S, Bisgaard H (2010) Long-term exposure to indoor air pollution and wheezing symptoms in infants. Indoor Air 20:159-167

11. Roda C, Guihenneuc-Jouyaux C, Momas I (2013) Environmental triggers of nocturnal dry cough in infancy: new insights about chronic domestic exposure to formaldehyde in the PARIS birth cohort. Environ Res 123:46-51

12. Rumchev KB, Spickett JT, Bulsara MK, Phillips MR, Stick SM (2002) Domestic exposure to formaldehyde significantly increases the risk of asthma in young children. Eur Respir J 20:403-406

13. Rumchev K, Spickett J, Bulsara M, Phillips M, Stick $S$ (2004) Association of domestic exposure to volatile organic compounds with asthma in young children. Thorax 59:746-751

14. Venn AJ, Cooper M, Antoniak M, Laughlin C, Britton J, Lewis SA (2003) Effects of volatile organic compounds, damp, and other environmental exposures in the home on wheezing illness in children. Thorax 58:955-960

15. Tavernier G, Fletcher G, Gee I, Watson A, Blacklock $G$, Francis $H$, Fletcher A, Frank T, Frank P, Pickering CA, Niven R (2006) IPEADAM study: indoor endotoxin exposure, family status, and some housing characteristics in English children. J Allergy Clin Immunol 117:656-662

16. Hwang G, Yoon C, Choi J (2011) A case-control study: exposure assessment of VOCs and formaldehyde for asthma in children. Aerosol Air Qual Res 11:908-914

17. Hulin M, Caillaud D, Annesi-Maesano I (2010) Indoor air pollution and childhood asthma: variations between urban and rural areas. Indoor Air 20:502-514

18. Hsu NY, Lee CC, Wang JY, Li YC, Chang HW, Chen CY, Bornehag CG, Wu PC, Sundell, Su HJ (2012) Predicted risk of childhood allergy, asthma, and reported symptoms using measured phthalate exposure in dust and urine. Indoor Air 22:186-199

19. Krzyzanowski M, Quackenboss JJ, Lebowitz MD (1990) Chronic respiratory effects of indoor formaldehyde exposure. Environ Res 52:117-125

20. Smedje G, Norbäck D, Edling C (1997) Asthma among secondary schoolchildren in relation to the school environment. Clin Exp Allergy 27:12701278

21. Garrett MH, Hooper MA, Hooper BM, Rayment PR, Abramson MJ (1999) Increased risk of allergy in children due to formaldehyde exposure. Allergy 54:330-337

22. Garrett MH, Rayment PR, Hooper MA, Abramson MJ, Hooper BM (1998) Indoor airborne fungal spores, house dampness and associations with environmental factors and respiratory health in children. Clin Exp Allergy 28:459-467

23. Jaakkola JJ, Parise H, Kislitsin V, Lebedeva NI, Spengler JD (2004) Asthma, wheezing, and allergies in Russian schoolchildren in relation to new surface materials in the home. Am J Public Health 94:560-562

24. Mi YH, Norbäck D, Tao J, Mi YL, Ferm M (2006) Current asthma and respiratory symptoms among pupils in Shanghai, China: influence of building ventilation, nitrogen dioxide, ozone, and formaldehyde in classrooms. Indoor Air 16:454-464

25. Zhao Z, Zhang Z, Wang Z, Ferm M, Liang Y,
Norbäck D (2008) Asthmatic symptoms among pupils in relation to winter indoor and outdoor air pollution in schools in Taiyuan, China. Environ Health Perspect 116:90-97

26. Kim J-L, Elfman L, Wieslander G, Ferm M, Toren K, Nordbäck D (2011) Respiratory health among Korean pupils in relation to home, school and outdoor environment. J Korean Med Sci 26:166-173

27. Martins PC, Valente J, Papoila AL, Caires I, Araújo-Martins J, Mata P, Lopes M, Torres S, Rosado-Pinto J, Borrego C, Annesi-Maesano I, Neuparth N (2012) Airways changes related to air pollution exposure in wheezing children. Eur Respir J 39:246-253

28. Wallner P, Kundi M, Moshammer H, Piegler K, Hohenblum P, Scharf S, Fröhlich M, Damberger B, Tappler P, Hutter HP (2012) Indoor air in schools and lung function of Austrian school children. J Environ Monit 14:1976-1982

29. Yeatts KB, El-Sadig M, Leith D, Kalsbeek W, Al-Maskari F, Couper D, Funk WE, Zoubeidi T, Chan RL, Trent CB, Davidson CA, Boundy MG, Kassab MM, Hasan MY, Rusyn I, Gibson JM, Olshan AF (2012) Indoor air pollutants and health in the United Arab Emirates. Environ Health Perspect 120:687-694

30. Annesi-Maesano I, Hulin M, Lavaud F, Raherison C, Kopferschmitt C, de Blay F, Charpin DA, Denis $C$ (2012) Poor air quality in classrooms related to asthma and rhinitis in primary schoolchildren of the French 6 Cities Study. Thorax 67:682-688

31. Dannemiller KC, Murphy JS, Dixon SL, Pennell KG, Suuberg EM, Jacobs DE, Sandel M (2013) Formaldehyde concentrations in household air of asthma patients determined using colorimetric detector tubes. Indoor Air 23:285-294

32. Zhai L, Zhao J, Xu B, Deng Y, Xu Z (2013) Influence of indoor formaldehyde pollution on respiratory system health in the urban area of Shenyang, China. Afr Health Sci 13:137-143

33. Madureira J, Paciencia I, Rufo J, Ramos E, Barros H, Teixeira JP, Fernandes EO (2015) Indoor air quality in schools and its relationship with children's respiratory symptoms. Atmos Environ 118:145-156

34. McGwin G, Lienert J, Kennedy Jl (2010) Formaldehyde exposure and asthma in children: a systematic review. Environ Health Perspect 118:313-317

35. Yao Y, Liang W, Zhu L, Duan Y, Jin Y, He L (2015) Relationship between the concentration of formaldehyde in the air and asthma in children: a meta-analysis. Int J Clin Exp Med 15:8358-8362

36. Norbäck D, Bjornsson E, Janson C, Widstrom J, Boman G (1995) Asthmatic symptoms and volatile organic compounds, formaldehyde, and carbon dioxide in dwellings. Occup Environ Med 52:388-395

37. Tarkowski M, Gorski P (1995) Increased IgE antiovalbumin level in mice exposed to formaldehyde. Int Arch Allergy Immunol 106:422-424

38. Sadakane K, Takano H, Ichinose T, Yanagisawa R, Shibamoto T (2002) Formaldehyde enhances mite allergen-induced eosinophilic inflammation in the murine airway. J Environ Pathol Toxicol Oncol 21:267-276

39. Jung WW, Kim EM, Lee EH, Yun HJ, Ju HR, Jeong MJ, Hwang KW, Sul D, Kang HS (2007) Formaldehyde exposure induces airway inflammation by increasing eosinophil infiltrations through the regulation of reactive oxygen species production. Environ Toxicol Pharmacol 24:174-182

40. Fujimaki H, Kurokawa Y, Kunugita N, Kikuchi M, Sato F, Arashidani K (2004) Differential immunogenic and neurogenic inflammatory responses in 
an allergic mouse model exposed to low levels of formaldehyde. Toxicology 197:1-13

41. Gu Y, Fujimiya Y, Kunugita N (2008) Long-term exposure to gaseous formaldehyde promotes allergen-specific lgE-mediated immune responses in a murine model. Hum Exp Toxicol 27:37-43

42. Liu D, Zheng Y, Li B, Yao H, Li R, Zhang Y, Yang X (2011) Adjuvant effects of gaseous formaldehyde on the hyper-responsiveness and inflammation in a mouse asthma model immunized by ovalbumin. J Immunotoxicol 8:305-314

43. Wu Y, You H, Ma P, Li L, Yuan Y, Li J, Ye X, Liu X, Yao H, Chen R, Lai K, Yang X (2013) Role of transient receptor potential ion channels and evoked levels of neuropeptides in a formaldehyde-induced model of asthma in BALB/c mice. PLoS ONE 8:e62827

44. Qiao Y, Li B, Yang G, Yao H, Yang J, Liu D, Yan Y, Sigsgaard T, Yang $X$ (2009) Irritant and adjuvant effects of gaseous formaldehyde on the ovalbumin-induced hyperresponsiveness and inflammation in a rat model. Inhal Toxicol 21:1200-1207

45. Riedel F, Hasenauer E, Barth PJ, Koziorowski A, Rieger CH (1996) Formaldehyde exposure enhances inhalative allergic sensitization in the guinea pig. Allergy 51:94-99

46. Swiecichowski AL, Long KJ, Miller ML, Leikauf GD (1993) Formaldehyde-induced airway hyperreactivity in vivo and ex vivo in guinea pigs. Environ Res 61:185-199

47. Nordman H, Keskinen H, Tuppurainen M (1985) Formaldehyde asthma - rare or overlooked? J Allergy Clin Immunol 75:91-99

48. Sheppard D, Eschenbacher WL, Epstein J (1984) Lack of bronchomotor response to up to $3 \mathrm{ppm}$ formaldehyde in subjects with asthma. Environ Res 35:133-139

49. Frigas E, Filley WV, Reed CE (1984) Bronchial challenge with formaldehyde gas: lack of bronchoconstriction in 13 patients suspected of having formaldehyde-induced asthma. Mayo Clin Proc 59:295-299

50. Sauder LR, Green DJ, Chatham MD, Kulle TJ (1987) Acute pulmonary response of asthmatics to $3.0 \mathrm{ppm}$ formaldehyde. Toxicol Ind Health 3:569-378

51. Green DJ, Sauder LR, Kulle TJ, Bascom R (1987) Acute response to $3.0 \mathrm{ppm}$ formaldehyde in exercising healthy nonsmokers and asthmatics. Am Rev Respir Dis 135:1261-1266

52. Uba G, Pachorek D, Bernstein J, Garabrant DH, Balmes JR, Wright WE, Amar RB (1989) Prospective study of respiratory effects of formaldehyde among healthy and asthmatic medical students. Am J Ind Med 15:91-101

53. Harving $\mathrm{H}$, Korsgaard J, Pedersen OF, Mølhave L, Dahl R (1990) Pulmonary function and bronchial reactivity in asthmatics during low-level formaldehyde exposure. Lung 168:15-21

54. Krakowiak A, Gorski P, Pazdrak K, Ruta U (1998) Airway response to formaldehyde inhalation in asthmatic subjects with suspected respiratory formaldehyde sensitization. Am J Ind Med 33:274-281

55. Casset A, Marchand C, Purohit A, le Calve $S$, Uring-Lambert B, Donnay C, Meyer P, de Blay F (2006) Inhaled formaldehyde exposure: effect on bronchial response to mite allergen in sensitized asthma patients. Allergy 61:1344-1350

56. Ezratty V, Bonay M, Neukirch C, Orset-Guillossou G, Dehoux M, Koscielny S, Cabanes PA, Lambrozo J, Aubier M (2007) Effect of formaldehyde on asthmatic response to inhaled allergen challenge. Environ Health Perspect 115:210-214
57. Wilhelmsson B, Holmström M (1987) Positive formaldehyde-RAST after prolonged formaldehyde exposure by inhalation. Lancet 8551:164

58. Kramps JA, Peltenburg LT, Kerklaan PR, Spieksma FT, Valentijn RM, Dijkman JH (1989) Measurement of specific lgE antibodies in individuals exposed to formaldehyde. Clin Exp Allergy 19:509-514

59. Grammer LC, Harris KE, Shaughnessy MA, Sparks P, Ayars GH, Altman LC, Patterson R (1990) Clinical and immunologic evaluation of 37 workers exposed to gaseous formaldehyde. J Allergy Clin Immunol 86:177-181

60. Grammer LC, Harris KE, Cugell DW, Patterson $R$ (1993) Evaluation of a worker with possible formaldehyde-induced asthma. J Allergy Clin Immunol 92:29-33

61. Dykewicz MS, Patterson R, Cugell DW, Harris KE, Wu AF (1991) Serum lgE and lgG to formaldehyde-human serum albumin: lack of relation to gaseous formaldehyde exposure and symptoms. J Allergy Clin Immunol 87:48-57

62. Lidén S, Scheynius A, Fischer T, Johansson SG, Ruhnek-Forsbeck M, Stejskal V (1993) Absence of specific lgE antibodies in allergic contact sensitivity to formaldehyde. Allergy 48:525-529

63. Wantke F, Focke M, Hemmer W, Tschabitscher M, Gann M, Tappler P, Götz M, Jarisch R (1996) Formaldehyde and phenol exposure during an anatomy dissection course: a possible source of IgE-mediated sensitization? Allergy 51:837-841

64. Wantke F, Focke M, Hemmer W, Bracun R, Wolf-Abdolvahab S, Götz M, Jarisch R, Götz M, Tschabitscher M, Gann M, Tappler P (2000) Exposure to formaldehyde and phenol during an anatomy dissecting course: sensitizing potency of formaldehyde in medical students. Allergy 55:84-87

65. Wantke F, Focke $M$, Hemmer W, Bracun R, Wolf-Abdolvahab S, Götz M, Jarisch R, Götz M, Tschabitscher M, Gann M, Tappler P (1996) Exposure to gaseous formaldehyde induces IgE mediated sensitization for formaldehyde in school-children. Clin Exp Allergy 26:276-280

66. Doi S, Suzuki S, Morishita M, Yamada M, Kanda Y, Torii S, Sakamoto T (2003) The prevalence of $\mathrm{lg}$ E sensitization to formaldehyde in asthmatic children. Allergy 58:668-671

67. Wolkoff P, Nielsen (2010) Non-cancer effects of formaldehyde and relevance for setting an indoor air guideline. Environ Int 36:788-799

68. Heinrich J (2011) Influence of indoor factors in dwellings on the development of childhood asthma. Int J Hyg Environ Health 214:1-25

69. Golden R (2011) Identifying an indoor air exposure limit for formaldehyde considering both irritation and cancer hazards. Crit Rev Toxicol 41:672-721

70. Nielsen GD, Larsen ST, Wolkoff P (2013) Recent trend in risk assessment of formaldehyde exposures from indoor air. Arch Toxicol 87:73-98

71. Tagiyeva N, Sheikh A (2014) Domestic exposure to volatile organic compounds in relation to asthma and allergy in children and adults. Expert Rev Clin Immunol 10:1611-1639

72. Nurmatov UB, Tagiyeva N, Semple S, Devereux G, Sheikh A (2015) Volatile organic compounds and risk of asthma and allergy: a systematic review. Eur Respir Rev 24:92-101

73. Kanchongkittiphon W, Gaffin JM, Phipatanakul W (2014) The indoor environment and inner-city childhood asthma. Asian Pac J Allergy Immunol 32:103-110

74. US-EPA (2010) Toxicological review of formaldehyde - inhalation assessment. In support of summary information on the Integrated Risk
Information System (IRIS). External review draft, Washington, DC. https://ofmpub.epa.gov/eims/ eimscomm.getfile?p_download_id=497038. Zugegriffen: 01.03.2016

75. ATSDR (1999) Toxicological profile for formaldehyde. Agency for Toxic Substances and Disease Registry, Atlanta, USA. http://www.atsdr.cdc.gov/ toxprofiles/tp.asp?id=220\&tid=39. Zugegriffen: März 2016

76. ATSDR (2010) Addendum to the toxicological profile for formaldehyde. Agency for Toxic Substances and Disease Registry,Atlanta, USA. US-EPA (2010) Toxicological review of formaldehyde - inhalation assessment. External review draft 2010. Integrated Risk Information System (IRIS). EPA/635/R-10/002 A. U.S. Environmental Protection Agency Washington DC, USA. http:// www.atsdr.cdc.gov/substances/toxsubstance. asp?toxid=39. Zugegriffen: März 2016

77. IOM (2000) Clearing the Air: Asthma and Indoor Air Exposures. Washington, DC: National Academies Press. http://www.nap.edu/catalog/9610/ clearing-the-air-asthma-and-indoor-air-exposures. Zugegriffen: März 2016

78. Kanchongkittiphon W, Mendel MJ, Gaffin JM, Wang G, Phipatankul W (2015) Indoor environmental exposures and exacerbation of asthma: an update of the 2000 review by the Institute of Medicine. Environ Health Perspect 123:6-20

79. Prenzel F (2009) Wheezing beim Säugling und Kleinkind. Kinder Jugendmed 6:340-348

80. Laußmann D, Haftenberger M, Langen U, Eis D (2012) Einflussfaktoren für Asthma bronchiale bei Kindern und Jugendlichen in Deutschland. Bundesgesundheitsblatt 55:308-317

81. Dales R, Raizenne M (2004) Residential exposure to volatile organic compounds and asthma. J Asthma 41:259-270

82. Le Cann P, Bonvallot N, Glorennec P, Deguen S, Goeury C, Le Bot B (2011) Indoor environment and children's health: recent developments in chemical, biological, physical and social aspects. Int J Hyg Environ Health 215:1-18

83. Kim KH, Jahan SA, Kabir E (2013) A review on human health perspective of air pollution with respect to allergies and asthma. Environ Int 59:41-52

84. Matsui EC (2014) Environmental exposures and asthma morbidity in children living in urban neighborhoods. Allergy 69:553-558

85. Sheehan WJ, Phipatanakul W (2015) Difficult-to-control asthma: epidemiology and its link with environmental factors. Curr Opin Allergy Clin Immunol 15:397-401

86. Wang IJ, Tung TH, Tang CS, Zhao ZH (2016) Allergens, air pollutants, and childhood allergic diseases. Int J Hyg Environ Health 219:66-71

87. Garcia GJ, Schroeter JD, Segal RA, Stanek J, Foureman GL, Kimbell JS (2009) Dosimetry of nasal uptake of water-soluble and reactive gases: a first study of interhuman variability. Inhal Toxicol 21:607-618

88. Ginsberg G, Foos B, Brown Dzubas R, Firestone M (2010) Options for incorporating children's inhaled dose into human health risk assessment. Inhal Toxicol 22:627-647

89. Franklin P, Dingle P, Stick S (2000) Raised exhaled nitric oxide in healthy children is associated with domestic formaldehyde levels. Am J Respir Crit Care Med 161:1757-1759 